\title{
Present status and technical directions of the EVN
}

\author{
M. Lindqvist ${ }^{* a}$, A. Szomoru ${ }^{b}$ \\ ${ }^{a}$ Department of Earth and Space Sciences, Chalmers University of Technology \\ Onsala Space Observatory, SE-439 92 Onsala, Sweden \\ ${ }^{b}$ Joint Institute for VLBI in Europe \\ Posbus 2, 7990 AA Dwingeloo, The Netherlands \\ E-mail: Michael.Lindqvist@chalmers.se, szomoru@jive.nl
}

\begin{abstract}
The European VLBI Network has evolved significantly during recent years. This has been made possible by the addition of new antennas, the introduction of the Mark 5 recording system and the emergence of high bandwidth optical fibre networks, the SFXC software correlator, and most recently, by the upgrade to digital backends at the stations. Here we describe the present status of the array and outline some of the planned future technical directions.
\end{abstract}

12th European VLBI Network Symposium and Users Meeting,

7-10 October 2014

Cagliari, Italy

${ }^{*}$ Speaker. 


\section{Introduction}

Very Long Baseline Interferometry (VLBI) is an important, and in many respects unique, observing method, with a strong synergy with e.g. the Low-Frequency Array (LOFAR) and the Square Kilometre Array (SKA). One of the major VLBI networks is the European VLBI Network (EVN). 15 radio astronomical institutes (including the Joint Institute for VLBI in Europe (JIVE)) in Europe, Asia, South Africa and the Americas form the EVN. It was created in 1980 and does not have any central funding. The EVN Consortium Board of Directors (CBD) determines the overall policy of the EVN. Astronomers who wish to use the EVN, must submit observing proposals to the EVN Programme Committee. Proposals are accepted from astronomers from all over the world (the EVN operates as an "open sky" observatory).

Another important body of the EVN is the Technical and Operations Group (TOG). It consists of personnel from the EVN stations (such as the VLBI friends, see below) and provides the technical and operational expertise for operating the EVN as a VLBI array. The TOG also advises the CBD on all aspects of technical and operational issues relevant to the reliability and performance of the network and implements technical and operational upgrades. In this paper we will describe the current status of the EVN and outline some of the future developments.

\section{Current status}

The EVN operates about 21 individual telescopes, which include some of the world's largest and most sensitive radio telescopes (ranging from 14-305 m), Figure 1. New telescopes includes the Yebes $40 \mathrm{~m}$ telescope in Spain and the Russian $32 \mathrm{~m}$ KVAZAR stations (Svetloe, Zelenchukskaya and Badary). They made their first EVN observations in 2008 and 2010, respectively. The most recent addition to the EVN family includes the Korean VLBI Network $21 \mathrm{~m}$ telescopes (Yonsei, Ulsan, Tamna), the Shanghai Tianma $65 \mathrm{~m}$ telescope. The Sardinia $64 \mathrm{~m}$ telescope is in a commissioning phase but will be available on a "best efforts" basis from 2015 on.

The EVN currently observes during 3 periods per year known as "EVN sessions". Each of these sessions is approximately 21 days long and typically involves 3-4 different observing frequencies (sometimes up to 5). The total amount of disk media used in an EVN session is currently $700-800 \mathrm{~TB}$ (the current record is $>1000 \mathrm{~TB}$ ). For a station participating in most users experiments this means a disk load of 50-60 TB. In addition, the EVN also offers ten 24 hour e-VLBI sessions per year, approximately once per month outside the main EVN sessions. The e-VLBI technique enables real-time data transfer from the stations to JIVE via optical fibre networks. It is also possible to propose for Out-of Session as well as Target-of Opportunity observing time. The scheduling is done by the EVN scheduler. The most common observing wavebands are 18 and $6 \mathrm{~cm}$. Other observing wavebands include $90,21,13,5,4,1$ and $0.7 \mathrm{~cm}$.

The maximum data rate is $1 \mathrm{Gbps}$ (corresponding to an observing bandwidth of $128 \mathrm{MHz}$, dual polarization, 2-bit sampling), both for disk recording and e-VLBI. Since 2014 Global VLBI (which adds the NRAO Very Long Baseline Array (VLBA) and/or other NRAO antennas) can be done at 1 Gbps (disk recording) as well.

The situation at the EVN stations is fairly diverse with respect to data-acquisition systems. However, all stations record the data using the Mark 5 system developed by the Haystack Ob- 


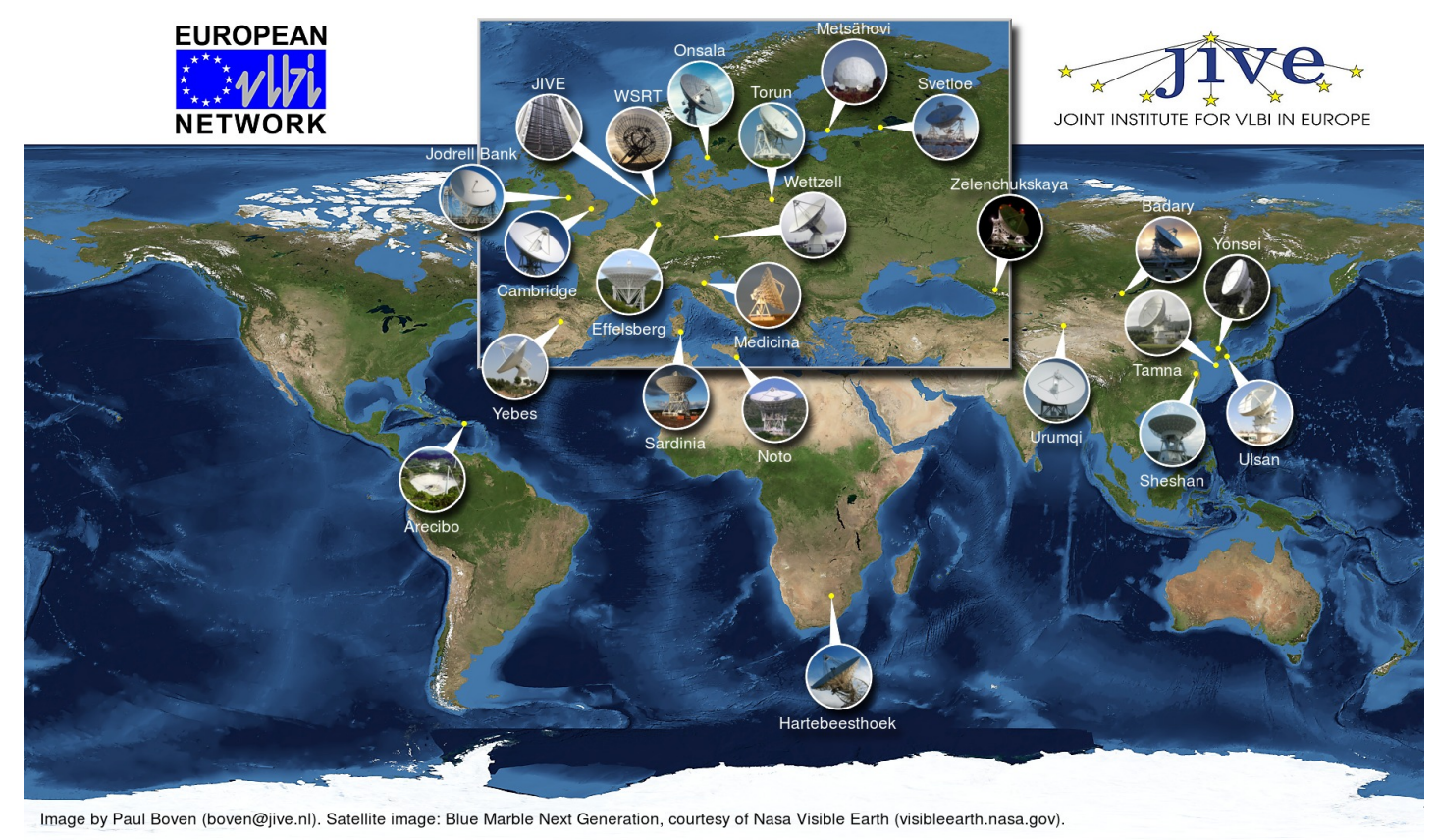

Figure 1: The European VLBI Network (credit: P. Boven, JIVE).

servatory ([13], [14]). Several stations are in the process of replacing (or have already replaced) their old analog data-acquisition systems (which are limited to $1 \mathrm{Gbps}$ ) with the Digital Base Band Converter (DBBC, [11]), the Russian KVAZAR stations have their own digital BBC called the R1002 ([3]), the Chinese stations use the Chinese VLBI Data Acquisition System CDAS ([16]), Arecibo uses the Haystack/NRAO Roach Digital Backend (RDBE, [8]) while Robledo has just started commissioning another digital BBC, developed by NASA, called the Deep Space Network VLBI Processor (DVP, [4]). All of these systems are capable of handling a recording rate of at least 2 Gbps (corresponding to an observing bandwidth of $256 \mathrm{MHz}$, dual polarization, 2-bit sampling).

The station VLBI friend is responsible for the EVN session at his/her station. The different components of a typical EVN session are shown in Figure 2. Each frequency block almost always starts with a Network Monitoring Experiment (NME). The purpose of the NME is to perform various tests of the network. The NME also includes a fringe test, which is extremely useful for correcting problems before the actual user experiments following the NME. For each frequency block the VLBI friend also checks and if necessary updates the gain curve and the system temperature calibration. These data are later stored in the so called ANTAB-file. The VLBI friend must aim to produce calibration information (i.e., the ANTAB-file) for each user experiment within 2 weeks after the end of an EVN session. For an e-VLBI session the VLBI friend should provide such data $<24$ hours after the experiment. The TOG typically meets every $8-9$ months to discuss recent sessions (here the feedback from JIVE to the stations is of utmost importance) as well as plans for the future. The accuracy of the EVN amplitude calibration (and how to improve it) is normally a major agenda point at the TOG-meetings.

Most of the data are correlated at JIVE but it is also possible to use the correlator at the MaxPlanck Institut für Radioastronomie in Bonn, Germany or the NRAO correlator in Soccorro, USA. 


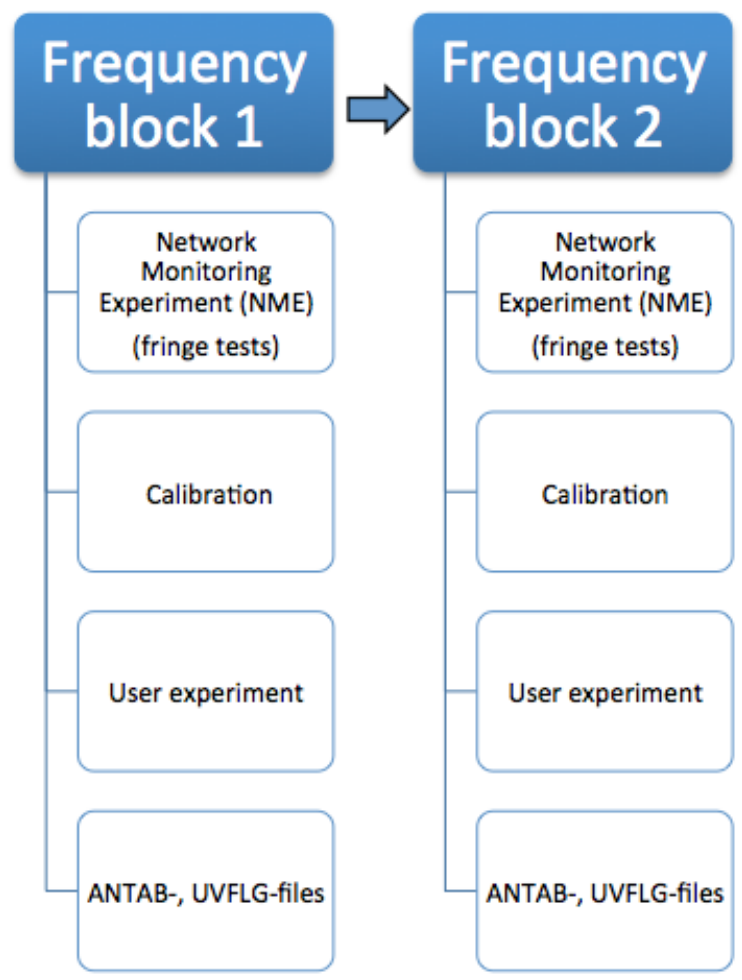

Figure 2: The components of a typical EVN session.

All of these correlators are software-based using either the EVN SFXC Correlator at JIVE (SFXC, [7]) or the Distributed FX (DiFX) software correlator ([1], [2]). In the rest of the paper we will only discuss the SFXC.

\section{Future possibilities}

Before investigating various technical possibilities it is interesting to start with the most precious assets of the network, the EVN users. They were asked to rank a series of items in the order based on their importance to his/her science during an evaluation of JIVE a few years ago. The results are given in Table 1. The average rank is an indication for the way the EVN users see the future of the EVN. When discussing future possibilities for the EVN, both in the TOG and the CBD, this must be taken into account.

\subsection{New telescopes and collaborations}

As can be seen in Table 1, the top priority for the EVN users is to have more telescopes and more shorter spacing. A natural way forward and currently under testing is to include e-Merlin with all its out-stations at $1 \mathrm{Gbps}$ (and beyond). It would also be very interesting to improve the uv-coverage towards the south (i.e., Hartebeesthoek, South Africa). Here the Africa VLBI Network $(\mathrm{AVN})$ is an interesting project aiming to convert old satellite telecommunications antennas found in many African countries ([5]). In the near future, we expect to add the $32 \mathrm{~m}$ radio telescope 


\begin{tabular}{cl}
\hline \hline Average rank & Item \\
\hline 2.73 & Improved uv-coverage (more telescopes, more short spacings) \\
3.50 & Increased bandwidth to improve sensitivity \\
4.57 & Improved calibration in general (phase, amplitude, bandpass, polarization) \\
4.60 & Improved resolution (more long baselines) \\
4.73 & Frequency agility for spectral index imaging \\
6.06 & Real-time e-VLBI capabilities for more telescopes \\
6.34 & Extended observing time to be able to carry out big surveys \\
6.42 & Real-time e-VLBI capabilities for a larger fraction of observing time \\
6.71 & Improved astrometry \\
6.89 & Larger field of view \\
\hline
\end{tabular}

Table 1: EVN capabilities and their ranking by EVN users.

at Irbene, operated by Ventspils International Radio Astronomy Center into the EVN operations. Further in the future we envision the collaboration with telescopes in China (such as the $50 \mathrm{~m}$ Miyun telescope, the $40 \mathrm{~m}$ Kunming telescope and the $500 \mathrm{~m}$ FAST), Meerkat in South Africa and the SKA ([9]).

EVN is not a full time array like the VLBA. This has led to a concept called "EVN light" where the smaller antennas operate as an array outside the major EVN sessions. Science areas for such a concept include e.g. long-term maser monitoring and fast automated response observations. If there is a sufficient interest from the user community the EVN should investigate in more detail how to operate such an array in terms of proposals, scheduling, correlation and user support. We see no major technical problems here.

\subsection{New observing modes}

In 2014 the EVN and the Long Baseline Array (LBA) in Australia announced the possibility to combine the arrays. The first attempt to truly combine EVN and LBA telescopes (and in fact telescopes in Australia, China, Europe, Japan, as well as North- and South-America) in a realtime e-VLBI experiment was done during a demo at the opening ceremony of the International Year of Astronomy in 2009. This was later followed up in a real science experiment [6]. The EVN also opened up the observing class Out-of-Session (OoS) observing (up to a maximum of 144 hours/year), for all projects during 2014. Up until then, OoS observing had been limited to EVN/Global+RadioAstron. Proposals requesting OoS observing time must provide full scientific (and technical if appropriate) justification as to why observations must be made outside regular EVN sessions.

Another observing class to be offered by the EVN will allow an observation to be scheduled automatically during an e-VLBI run if a specific set of triggering criteria is met (e.g. through a VOEvent (a standardized language used to report astronomical events) or other form of automated/manual trigger response). The expected response time to execute a new program is about 10 minutes. The station experiment setup will be the same as the interrupted program. Source coordinates need only be included in the trigger request, not in the original proposal. This is the first time the EVN considers generic triggers. 
NEXPReS was an e-Infrastructure project led by JIVE and funded by the European Union's Seventh Framework Programme. The project successfully concluded on 30 June 2013. One of the aims of NEXPReS was to introduce an e-VLBI element into all EVN operations, to take away the distinction between recorded and real-time VLBI. Although this has not quite happened in practice, it now is certainly feasible. Thus, there are no real technical obstacles to doing disk recording and e-VLBI simultaneously even at 4 Gbps.

\subsection{Next generation backends and recording systems}

With more and more EVN stations acquiring and starting to use digital BBCs, and the VLBA switching to the exclusive use of RDBE, the prospect of doing astronomical VLBI at 2 and 4 Gbps on a regular base has become quite realistic. In fact, the VLBA started limited 2 Gbps operation already in February 2012 and is now fully operational. Presently, the EVN operates at a maximum of $1 \mathrm{Gbps}$ and the current plan is to offer 2 Gbps (disk recording) in 2015. Most likely we will have an array where a few stations still observe at $1 \mathrm{Gbps}$ and the rest at $2 \mathrm{Gbps}$ (mixed mode observations). Correlation of such data has been successfully tested using the SFXC. Increasing the data rate means of course that the EVN needs to increase its disk pool which already today limits the number of projects that can be scheduled. However, the cost is relatively small compared to the total cost of running the EVN.

The next step is to upgrade the EVN (or at least some of the stations) to 4 Gbps (disk recording) but there is no firm plan when this should be implemented. This implies a bandwidth of $512 \mathrm{MHz}$ (dual polarization and 2-bit sampling) and one issue here is to find a common frequency range (which may be the case also for $2 \mathrm{Gbps}$ ) at some wavebands.

The EVN has not really discussed observations beyond 4 Gbps. However, one could envision operations above that at the higher frequency bands ( 22 and $43 \mathrm{GHz}$ ), in particular since the number of telescopes in the EVN capable of operating at $22 \mathrm{GHz}$ (and beyond) has increased substantially in the past years. With the third generation of the DBBC, the DBBC3 ([12]), it will be possible to operate at $32 \mathrm{Gbps}$ ( $4 \mathrm{GHz}$ bandwidth, dual polarization and 2-bit sampling).

The EVN will move towards diskshipping-less operation using either a FlexBuff or a Mark 6. The FlexBuff was designed at Metsähovi Finland as part of the NEXPReS project. It is basically a server with a big, non-shippable box of hard disks, with clever software optimized towards very high-speed simultaneous recording/playback. The Mark 6 system is being developed at MIT Haystack ([15]). It uses the same chassis as the previous Mark 5 versions (A, A+, B, B+ and C). Apart from the enclosures, disk modules and a custom-made backplane, it is fully Commercial-offthe-shelf (COTS), and all software is open source. The Mark 6 comes in two versions, one capable of $8 \mathrm{Gbps}$ recording on two disk packs, and a $16 \mathrm{Gbps}$ version. The latter version records onto four disk packs, two of which are mounted in a second enclosure.

Turning to diskshipping-less operations would have many significant advantages. Stations would not need to bother with conditioning, changing and shipping disk packs, transfers could be done practically automatically, results of a significant sub-set of the EVN network that would be available in real-time, no packs would need to be sent out before each session. Unfortunately, some stations do not have sufficient connectivity (for example, the KVAZAR or the Chinese stations). It is not likely either that e-shipping will become a viable option for the VLBA (i.e., for Global VLBI project) anytime soon. 


\section{4 e-VLBI}

The maximum data rate for e-VLBI is currently 1 Gbps. Even if most EVN stations can operate at 1 Gbps there are still important stations that are not connected, notably the KVAZAR stations and Urumqi. The main limiting factor to operate beyond $1 \mathrm{Gbps}$ is thus the connectivity even though many stations do have excellent connections (10 Gbps). The National Research and Education Networks (NRENs) across Europe are being upgraded to a capacity of 40/100 Gbps and it is likely that many EVN stations will follow. As investigated in NEXPReS, the EVN could make use of Bandwidth on Demand (BoD) which is being implemented by the NRENs. BoD is a service that would allow a customer such as the EVN to request bandwidth at desired level when and where it need it, e.g. during an e-VLBI session.

The current SFXC cluster at JIVE can handle 13 stations at 1 Gbps. 16 stations at $4 \mathrm{Gbps}$ would mean a factor of $\sim 5$ more hardware. Another (less costly) possibility would be to use the FPGA based UniBoard correlator ([10]), see below. 4 UniBoards can handle 16 stations at 4 Gbps. One board is roughly equivalent to the old MarkIV EVN correlator.

\subsection{The correlators at JIVE}

The SFXC software correlator ([7]), developed and maintained at the JIVE has been used for all EVN correlation since December 2012 (the old MarkIV hardware correlator has not been used since then). It has opened up novel modes such as, mixed bandwidth correlation, pulsar gating, possibility to combine gating and binning, (practically) unlimited spectral resolution, wide-field imaging by using multiple phase centres (under development also in real time) and a phased array mode.

Uniboard ([10]) was a Joint Research Activity in RadioNet FP7 (a project supported by the European Commission under the 7th Framework Programme) led by JIVE. The aim was to create a generic high performance computing platform for radio astronomy. One application is the JIVE UniBoard Correlator (JUC).

The main advantage using the software correlator approach is that it is fantastically flexible. It is easy to modify, improve, extend, expand and upgrade. Furthermore, hardware gets cheaper as time goes by. One should however not underestimate the effort need to make it into an operational system. In addition, it is not very well suited for heavy lifting such as e-VLBI at 4 Gbps. On the other hand, the JUC will be perfect for simple operations (such as standard 4 Gbps experiments). The disadvantages of the JUC are that it is not nearly as flexible as a software correlator and it is much harder to develop firmware. One could envision that JIVE will operate 2 correlators, both the SFXC and the JUC.

\section{Conclusions}

As has been shown at this conference, the EVN is producing excellent science. This is of course of fundamental importance and the main motivation for further developments of the EVN. In order to meet the demands of the astronomers the EVN is making use of and driving technical progress. We have shown in this paper that much of the instrumentation needed to take the next steps already exists today, both at the stations and at JIVE. With its unique capabilities, which will complement the SKA as it is being rolled out, the future of the EVN is definitely looking bright. 


\section{References}

[1] A.T. Deller, S.J. Tingay, M. Bailes, C. West, DiFX: A Software Correlator for Very Long Baseline Interferometry Using Multiprocessor Computing Environments, PASP, 119, 318, 2007.

[2] A.T. Deller, W.F. Brisken, Phillips C.J., et al., DiFX-2: A More Flexible, Efficient, Robust, and Powerful Software Correlator, PASP, 123, 275, 2011.

[3] L. Fedotov, E. Nosov, S. Grenkov, D. Marshalov, The Digital Data Acquisition System for the Russian VLBI Network of New Generation, in IVS 2010 General Meeting Proceedings, edited by D. Behrend and K.D. Baver, 400, 2010.

[4] C. García-Miró, S.P. Rogstad, R. Navarro, et al., VLBI Data Acquisition Terminal Modernization at the Deep Space Network, in IVS 2012 General Meeting Proceedings, edited by D. Behrend and K.D. Baver, 171, 2012.

[5] M.J. Gaylard, M.F. Bietenholz, L. Combrinck, et al., An African VLBI network of radio telescopes in Proceedings of SAIP2011, the 56th Annual Conference of the South African Institute of Physics, edited by I. Basson and A.E. Botha, 473, 2011.

[6] M. Giroletti, Z. Paragi, H. Bignall, et al., Global e-VLBI observations of the gamma-ray narrow line Seyfert 1 PMN J0948+0022, A\&A, 528, L11, 2011.

[7] A. Keimpema, M.M. Kettenis, S.V. Pogrebenko, et al., The SFXC software correlator for Very Long Baseline Interferometry: Algorithms and Implementation, Experimental Astronomy, in press, arXiv:1502.00467.

[8] A. Niell, M. Bark, C. Beaudoin, et al., RDBE Development and Progress, in IVS 2010 General Meeting Proceedings, edited by D. Behrend and K.D. Baver, 396, 2010.

[9] Z. Paragi, L. Godfrey, C. Reynolds, et al., Very Long Baseline Interferometry with the SKA, in Advancing Astrophysics with the Square Kilometre Array, in press, arXiv: 1412.5971.

[10] A. Szomoru, The UniBoard, in Proceedings of the 10th European VLBI Network Symposium and EVN Users Meeting: VLBI and the new generation of radio arrays, POS (10th EVN Symposium) 098 , 2010.

[11] G. Tuccari, W. Alef, A. Bertarini, et al., DBBC2 Backend: Status and Development Plan, in IVS 2010 General Meeting Proceedings, edited by D. Behrend and K.D. Baver, 392, 2010.

[12] G. Tuccari, DBBC3 - A Full Digital Implementation of the VLBI2010 Backend, in IVS 2012 General Meeting Proceedings, edited by D. Behrend and K.D. Baver, 76, 2012.

[13] A. Whitney, Disk-Based VLBI Recording, in Future Directions in High Resolution Astronomy: The 10th Anniversary of the VLBA, ASP Conference Proceedings, San Francisco: Astronomical Society of the Pacific, Edited by J. Romney and M. Reid, 340, 588, 2005.

[14] A. Whitney, C. Ruszczyk, J. Romney, K. Owens, The Mark 5C VLBI Data System, in IVS 2010 General Meeting Proceedings, edited by D. Behrend and K.D. Baver, 373, 2010.

[15] A. Whitney, D. Lapsley, Mark 6 Next-Generation VLBI Data System, in IVS 2012 General Meeting Proceedings, edited by D. Behrend and K.D. Baver, 86, 2012.

[16] R. Zhu, X. Zhang, W. We, et al., The Progress of CDAS, in IVS 2010 General Meeting Proceedings, edited by D. Behrend and K.D. Baver, 388, 2010. 